Textures and Microstructures, 1989, Vol. 10, pp. 195-209

Reprints available directly from the publisher

Photocopying permitted by license only

(C) 1989 Gordon and Breach Science Publishers Inc.

Printed in the United Kingdom

\title{
Analytic Prediction of Texture and Length Changes During Free-End Torsion
}

\author{
L. S. TÓTH, † and J. J. JONAS
}

Department of Metallurgical Engineering, McGill University, 3450 University Street, Montreal, H3A 2A7 Canada

(Received 29 August, 1988)

The free-end torsion length change measurements of Rose and Stüwe (1968), carried out on cube-textured $\mathrm{Cu}$ tubes, are reanalyzed. Ratios of length change rate to applied shear rate are calculated using a newly-developed rate sensitive approach and also by the CMTP method. The dependence of this ratio on the orientation of the cube axis is shown to be in good agreement with the measurements, particularly for lengthening. The analysis is extended to its rate insensitive limit for comparison with the equivalent predictions of Rose and Stüwe. The rate of lattice rotation is also predicted; this is shown to be independent of rate sensitivity and identical to the rigid body rotation rate. Through the lattice rotations, analytic expressions are derived which predict the dependence of sample length on shear strain. These are also in good agreement with the experimental observations.

KEY WORDS: Plastic deformation, strain rate sensitivity, model calculations, length changes, texture changes, torsion testing.

\section{INTRODUCTION}

In 1968, Rose and Stüwe published some experimental results obtained from the free end torsion testing of a series of specially prepared thin-walled copper tubes. These has been fabricated from a sheet having a strong initial cube texture, and were constructed so

† On leave from Institute for General Physics, Eötvös University, 1445. Budapest, P.O.B. 323, Hungary 
that the cube axes in the sheet were inclined at increasing angles to the axial direction of the tube. In this way, they were able to provide the first explanation for the link between the length change rate in free and torsion and the starting orientation of the tube, i.e. the texture. Their calculation was based on the Schmid law and treated the sample as consisting essentially of one single crystal. Somewhat better agreement with their experimental results was later obtained by Lequeu et al. (1985) using the continuum mechanics of textured polycrystals ('CMTP') method. However, they treated the above case as if it involved a solid bar in terms of the imposed constraints. More recently, the present authors have shown (Tóth, Jonas, Gilormini, Bacroix, in press) that the free end torsion of thin walled tubes can be readily simulated by employing a Sachs type of approach which is suitably modified to account for the rate sensitivity of slip. In this paper, this rate sensitive model is applied first to calculate the initial rate of length change, then the orientation change during plastic straining is also calculated. In this way, the length changes occurring during shear deformation are derived entirely analytically as a function of the starting orientation. The results obtained show good agreement with the experimental results of Rose and Stüwe.

\section{CMPT VERSUS RATE SENSITIVITY}

The samples in the experiments we wish to simulate were polycrystals. Because of their sharp textures, they can also be considered as slightly disoriented single crystals. Now the main difference between the yield surface of a perfect single crystal and that of a disoriented crystal involves the rounded nature and lack of sharp edges of the latter. Thus our simulation will te based on smoothed yield surfaces, the existence of which was the justification for the development of the CMTP method by Montheillet et al. (1985) and Lequeu and coworkers $(1985,1987 a, b, 1988)$. One disadvantage of their approach, however, is that orientation changes cannot be calculated. By contrast, by employing the rate sensitivity of slip, the yield surface (or more exactly, the yield potential) remains smooth, as in the CMTP method, but in this case the lattice rotation rate can be predicted as well (see Tóth, Gilormini, Jonas, 1988, for example). 
This is the approach that is described below. A further advantage is that the analytic solutions obtained in this way can be extrapolated to the rate insensitive limit, as will be shown below, for comparison with the results derived by classical (rate insensitive) methods. Finally, the results of the present analysis are compared with those obtained from the CMTP approach in Section 6.

\section{INITIAL ORIENTATIONS AND BOUNDARY CONDITIONS}

In Rose and Stüwe's experiments, the starting material was an annealed thin copper sheet containing a strong cube texture (with a spread of about 5 degrees s.d. about the ideal orientation). Tubes were then formed by cutting out samples of different orientations from the sheet, each defined by the angle $\alpha$ between the longitudinal (2) axis of the tube and the [010] axis of the sheet, see Figure 1. Finally, torsion tests were performed on the tubes under conditions of free end testing up to strains of $Y \approx 0.15$. From the above it follows that the transformation from the sample to the crystal system is a simple rotation around the (3) axis of the specimen:

$$
T=\left[\begin{array}{ccc}
\cos \alpha & \sin \alpha & 0 \\
-\sin \alpha & \cos \alpha & 0 \\
0 & 0 & 1
\end{array}\right]
$$

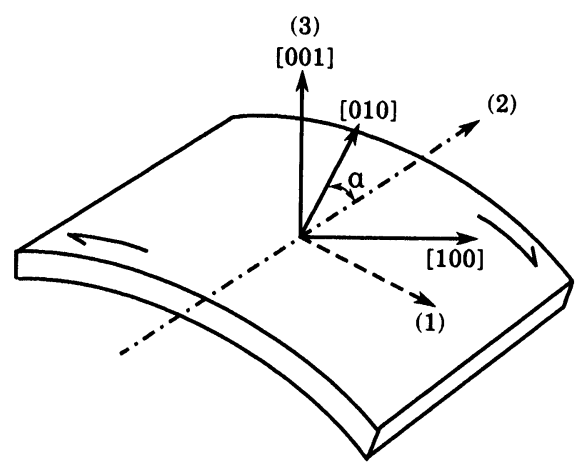

Figure 1 The configurations of the sample (1-2-3) and crystal $\langle 100\rangle$ reference systems. 
The boundary condition for the stresses in the case of tube testing is that the surface tractions at the inner and outer surfaces must vanish. For a thin walled tube, this condition leads to the plausible assumption that the corresponding stress component $\left(\sigma_{33}\right)$ is also zero within the tube wall. As no traction is applied along the axis of the tube (free end condition), $\sigma_{22}$ also vanishes. As a consequence, the third normal component, $\sigma_{11}$, must vanish as well, because of equilibrium requirements. This is the basis for the assumption that the stress state in the sample system is fairly accurately given by:

$$
\sigma=\left[\begin{array}{lll}
0 & \tau & 0 \\
\tau & 0 & 0 \\
0 & 0 & 0
\end{array}\right]
$$

\section{RATE-SENSITIVE SOLUTION}

The rate sensitivity of slip is expressed in the usual power law form:

$$
\dot{\Upsilon}_{s}=\frac{\dot{\Upsilon}_{0}}{\tau_{0}^{1 / m}} \tau_{s}\left|\tau_{s}\right|^{1 / m-1}
$$

where $\dot{Y}_{0}$ and $\tau_{0}$ are reference constants, $m$ is the rate sensitivity exponent, and $\tau_{s}$ is the resolved shear stress acting on the slip system identified by the index $s$. In the sample system, $\tau_{s}$ can be expressed as

$$
\tau_{s}=\tau\left(m_{12}^{s}+m_{21}^{s}\right)
$$

where $m_{i j}^{s}=b_{i}^{s} n_{j}^{s}$ ( $b$ is the slip direction and $n$ is the slip plane normal, see Tóth, Gilormini, Jonas, 1988 for the values of $m_{i j}^{s}$ ). Using the transformation given by Eq. (1) together with Eqns. 2 and 4 , the resolved shear stresses on the twelve fcc systems are the following:

$$
\begin{gathered}
\tau_{1}=\tau_{10}=-\tau_{5}=-\tau_{8}=\tau(\cos 2 \alpha-\sin 2 \alpha) / \sqrt{ } 6 \\
\tau_{2}=\tau_{4}=\tau_{7}=\tau_{11}=\tau(\cos 2 \alpha+\sin 2 \alpha) / \sqrt{ } 6 \\
\tau_{3}=\tau_{6}=\tau_{9}=\tau_{12}=\tau(2 \sin 2 \alpha) / \sqrt{ } 6
\end{gathered}
$$

The application of our constitutive law (Eq. 3) together with Eqs. 
$(5 \mathrm{a}-\mathrm{c})$ leads to the following relations between the shear rates:

$$
\begin{gathered}
\dot{\Upsilon}_{1}=\dot{\Upsilon}_{10}=-\dot{\Upsilon}_{5}=-\dot{\Upsilon}_{8} ; \quad \dot{\Upsilon}_{2}=\dot{\Upsilon}_{4}=\dot{\Upsilon}_{7}=\dot{\Upsilon}_{11} ; \\
\dot{\Upsilon}_{3}=\dot{\Upsilon}_{6}=\dot{\Upsilon}_{9}=\dot{\Upsilon}_{12} .
\end{gathered}
$$

These relations indicate that, out of the full set of twelve slip systems, there are three distinct subsets, within each of which four systems are deforming at identical shear rates. The assumption regarding the stress state (Eq. 2) means that the components of the strain rate tensor are all free, except for $\dot{\varepsilon}_{12}=\dot{\varepsilon}_{21}=\dot{\Upsilon} / 2$ (the latter are fixed by the imposed shear rate $\dot{Y}$ ). All the elements of the strain rate tensor can now be calculated using Eqs. (3) and (5) through the relation:

$$
\dot{\varepsilon}_{i j}=\frac{1}{2} \sum_{s=1}^{12}\left(m_{i j}^{s}+m_{j i}^{s}\right) \dot{\Upsilon}_{s} .
$$

The analysis leads to the result that the only non-zero strain rate components are $\dot{\varepsilon}_{11}=-\dot{\varepsilon}_{22}$ and $\dot{\varepsilon}_{12}=\dot{\Upsilon} / 2$. Thus there is no change in wall thickness, a result which was also obtained in simulations of the free end torsion of polycrystalline thin walled tubes (Tóth, Jonas, Gilormini, Bacroix in press). The ratio of length change rate to applied shear rate is:

$\frac{\dot{\varepsilon}}{\dot{\Upsilon}}=\frac{1}{2}$

$$
\times \frac{\cos 4 \alpha\left\{|\cos 2 \alpha-\sin 2 \alpha|^{1 / m-1}-\mid \cos 2 \alpha\right.}{|\cos 2 \alpha-\sin 2 \alpha|^{1 / m+1}+|\cos 2 \alpha+\sin 2 \alpha|^{1 / m+1}+|2 \sin 2 \alpha|^{1 / m+1}} .
$$

For the Newtonian viscous $(m=1)$ case:

$$
\frac{\dot{\varepsilon}}{\dot{\Upsilon}}=-\frac{1}{2} \frac{\sin 4 \alpha}{1+2 \sin ^{2} 2 \alpha} \text {. }
$$

In Figure 2, $\dot{\varepsilon} / \dot{\Upsilon}$ is plotted as a function of $\alpha$ for $m$-values of 0.05 , 0.167 and 1 . As can be seen, the curves are symmetrical with respect to $\alpha=45^{\circ}$. It can also be verified from Eq. (8) that the strain rate ratio $\dot{\varepsilon} / \dot{Y}$ does not depend on $m$ at $\alpha=(\arcsin$ $1 / \sqrt{ } 10) / 2=9.22^{\circ}$ and $\alpha=22.5^{\circ}$, at which its value is -0.25 . 


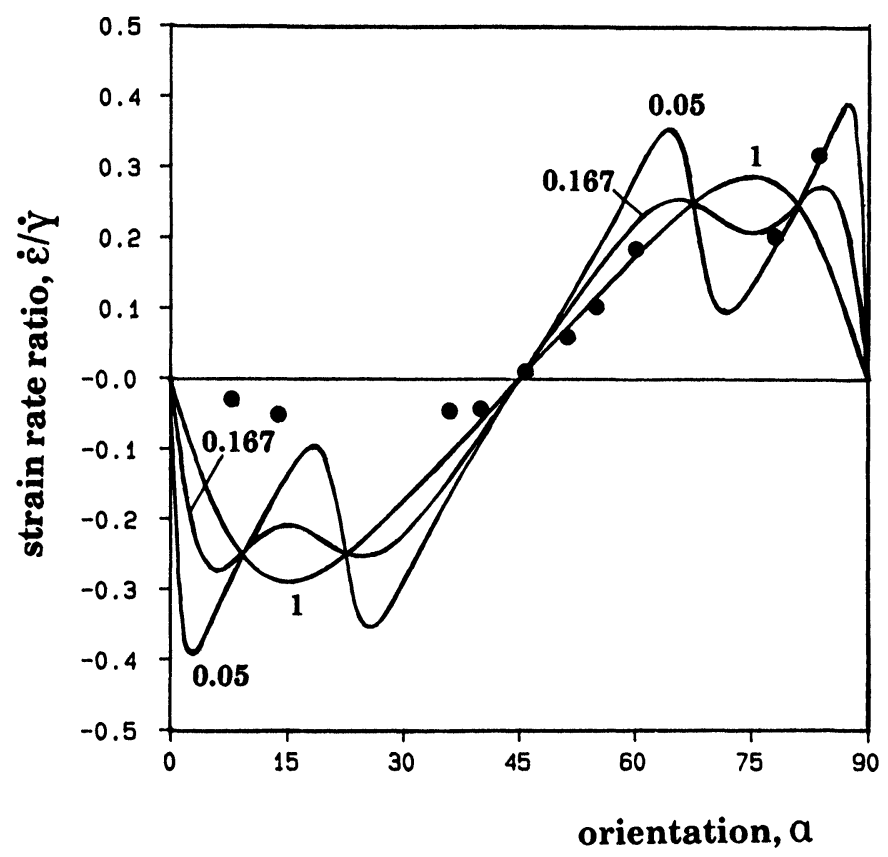

Figure 2 Ratio of the length change rate $\dot{\varepsilon}$ to the applied shear rate $\dot{Y}$ as a function of orientation. The continuous curves correspond to the rate sensitivities indicated. The full circles are the experimental points reported by Rose and Stüwe (1968).

In Figure 2, full circles denote the experimental points measured by Rose and Stüwe (1986). There is relatively good agreement between the predicted and measured values, especially for the case of lengthening. The curves corresponding to $m=0.167$ and $m=1$ are equally close to the experimental points. On the right hand side of the diagram, the agreement is only slightly less satisfactory for the $m=0.05$ case.

It should be pointed out that, in the absence of core splitting or other asymmetric glide effects, lengthening and shortening can be produced in the same samples, and at the same rate, by simply reversing the direction of twisting (i.e. from clockwise to counterclockwise and vice versa) (Semiatin et al. 1985). Such reversibility is equivalent to the symmetry in Figure 2 about $45^{\circ}$. Accordingly, the 
view is taken here that, given the considerable difficulties involved in making the samples and carrying out free end torsion tests, the data corresponding to shortening have been affected by experimental problems of an unknown nature.

\section{RATE-INSENSITIVE LIMIT}

In order to compare the predictions of the present rate sensitive analysis with Rose and Stüwe's rate insensitive predictions, the limiting $m=0$ solution will now be derived. In the rate sensitive case, all 12 slip systems are generally active. By contrast, in the rate-insensitive (Bishop and Hill) case, the number of activated systems is reduced to between 1 and 8 . From Eqs. (5a-c), the operating slip systems can be obtained by comparing the absolute values of the $\tau_{s}$ 's. There are three different values of $\tau_{s}$, which are plotted in Figure 3 as a function of $\alpha$. We find the following three intervals:

(i) $0<\alpha<22.5^{\circ}$

$\tau_{2}$ is maximum; systems: $2,4,7,11$

(ii) $22.5^{\circ}<\alpha<67.5^{\circ} \quad \tau_{3}$ is maximum; systems: $3,6,9,12$

(iii) $67.5^{\circ}<\alpha<90^{\circ} \quad-\tau_{1}$ is maximum; systems: $-1,-10,5,8$

Because of the rate-sensitive constitutive law (Eq. 3), in the rate-insensitive limit, only the maximally activated slip systems can operate. As the shear rate is governed by an exponential relation, any slip system which is not maximally activated carries a zero shear rate in the rate-insensitive limit. The limiting solutions obtained in this way are the following:

$$
\begin{gathered}
\text { Interval } 0<\alpha<22.5^{\circ}: \quad \frac{\dot{\varepsilon}}{\dot{\Upsilon}}=-\frac{1}{2} \frac{\cos 4 \alpha}{1+\sin 4 \alpha} \\
\text { Interval } 22.5^{\circ}<\alpha<67.5^{\circ}: \quad \frac{\dot{\varepsilon}}{\dot{\Upsilon}}=-\frac{1}{2 \operatorname{tg} 2 \alpha} \\
\text { Interval } 67.5^{\circ}<\alpha<90^{\circ}: \quad \frac{\dot{\varepsilon}}{\dot{\Upsilon}}=\frac{1}{2} \frac{\cos 4 \alpha}{1-\sin 4 \alpha}
\end{gathered}
$$

The curves corresponding to the above relations are plotted in Figure 4. The results obtained are similar to the predictions of Rose 


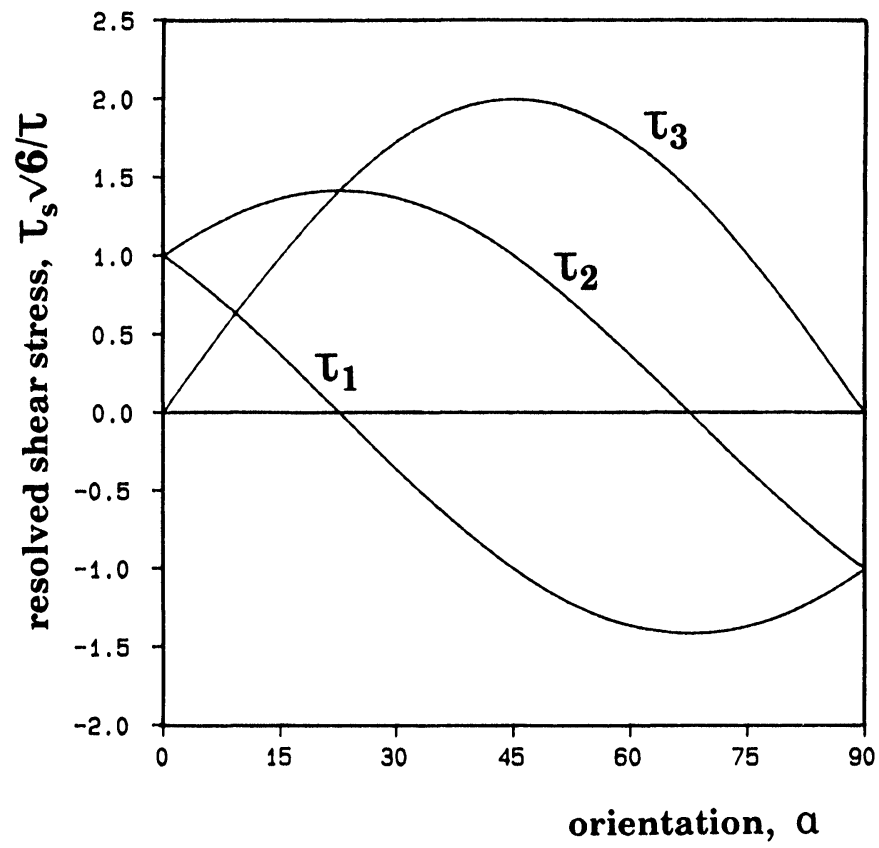

Figure 3 The resolved shear stress (normalized by the applied shear stress) as a function of orientation.

and Stüwe (1968), but differ by a factor of 2, for which no explanation is offered here. Eqs. $(10 \mathrm{a}-\mathrm{c})$ are not valid at $\alpha=22.5^{\circ}$ and $67.5^{\circ}$. As discussed in the previous section, in these cases, the rate sensitive result is independent of $m$; as a consequence, the limiting solutions are $\dot{\varepsilon} / \dot{Y}=-0.25$ and 0.25 , respectively. It should be added here that the $m=0$ curves of Figure 4 provide a satisfactory explanation for the somewhat unexpected shapes of the $m=0.05$ curves introduced in Figure 2 .

\section{CMTP SOLUTION}

In the present case, the stress state is completely defined by Eq. (2). As a consequence, the strain rate vector normal to the CMTP yield 


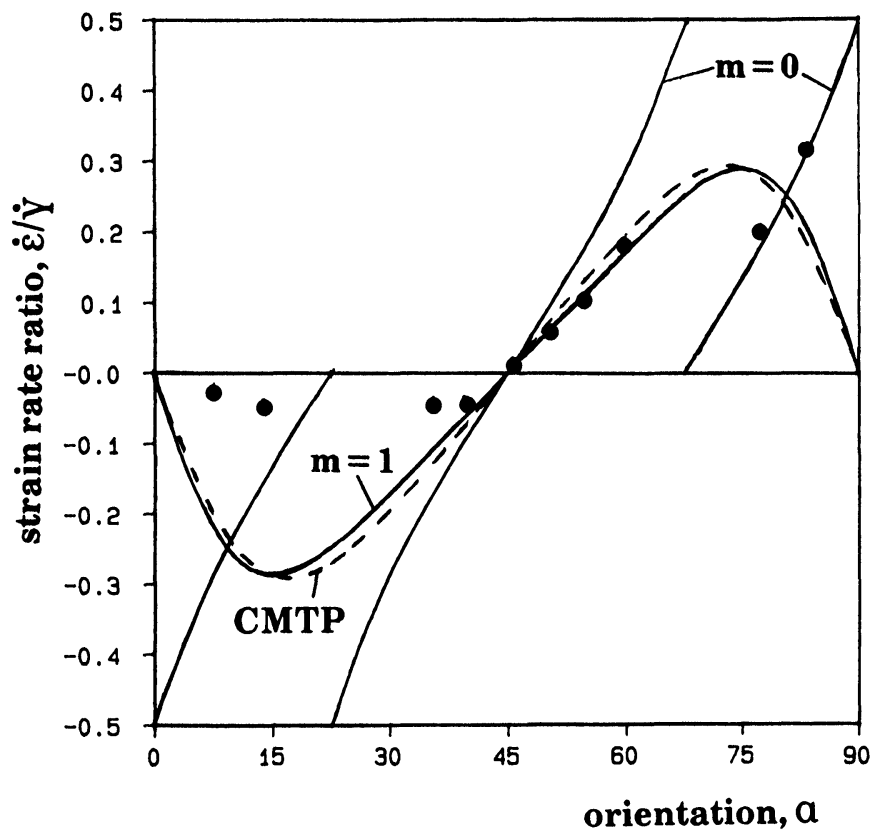

Figure 4 Comparison of the predictions for linear rate sensitivity $(m=1)$ with those of the CMTP method and the limiting $m=0$ solutions.

surface at this point can be readily derived. We use the following (quadratic) CMTP yield function (Lequeu et al. 1985):

$f=\frac{3}{2}\left(S_{11}^{2}+S_{22}^{2}+S_{33}^{2}\right)+\frac{2}{3}\left(S_{12}^{2}+S_{21}^{2}+S_{23}^{2}+S_{32}^{2}+S_{13}^{2}+S_{31}^{2}\right)=1$

where the $S_{i j}$ 's are the deviatoric stress components and the unit of stress is $\sqrt{ } 6 \tau_{c}$. The strain rate tensor is found by transforming $\sigma$ into the crystal system and using the normality rule together with Eq. (11). For the strain rate ratio $\dot{\varepsilon} / \dot{Y}$, we obtain:

$$
\frac{\dot{\varepsilon}}{\dot{\Upsilon}}=-\frac{7 \sin 4 \alpha}{36-20 \cos ^{2} 2 \alpha} \text {. }
$$

For comparison, the $m=1$ solution from Eq. (9) is:

$$
\frac{\dot{\varepsilon}}{\dot{Y}}=-\frac{6 \sin 4 \alpha}{36-24 \cos ^{2} 2 \alpha} \text {. }
$$


As can be seen from Eqs. (12) and (13), the linear viscous and CMTP solutions are very close; see the two curves in Figure 4. In the work by Lequeu et al. (1985), the boundary condition for the free end torsion of solid bars was employed for the CMTP solution, which is why they obtained smaller values for $\dot{\varepsilon} / \dot{Y}$ (Lequeu et al. 1985).

\section{CALCULATION OF the ORIENTAtion change}

In what follows, the rate of orientation change is calculated by assuming that the orientations of the shear plane normal (axis 2) and the shear direction (axis 1) remain fixed. These conditions lead to the following components of the lattice rotation rate (Tóth, Jonas, Gilormini, Bacroix in press, Tóth, Gilormini Jonas, 1988):

$$
\dot{\Omega}_{12}=\sum_{s} m_{21}^{s} \dot{\Upsilon}^{s}, \quad \dot{\Omega}_{13}=\sum_{s} m_{31}^{s} \dot{\Upsilon}^{s}, \quad \dot{\Omega}_{32}=\sum_{s} m_{23}^{s} \dot{\Upsilon}^{s} .
$$

Using Eqs. (3), (5) and (6), it can readily be shown that $\dot{\Omega}_{13}=\dot{\Omega}$ ${ }_{32}=0$ and $\dot{\Omega}_{12}=\dot{Y} / 2$. This means that the rate of orientation change is constant and takes place solely around the specimen 3 axis; furthermore, it is independent of the rate sensitivity exponent. As the lattice rotation rate is the difference between the rigid body and plastic spins (see e.g. Refs. (Tóth, Jonas, Gilormini, Bacroix in press, Aifantis 1987, Canova et al. 1988)), this means that the plastic spin vanishes for all the orientations in question and that the rate of orientation change is equal to the rigid body rotation rate. This result is interesting because zero plastic spin for torsional deformation was only obtained previously for the $m=1$ case (Tóth, Gilormini, Jonas, 1988, Canova et al. 1988, Harren, et al. in press). By contrast, for the oriented cube textures considered here, the plastic spin rate is zero at all values of rate sensitivity (as well as in the rate-insensitive limit).

\section{ANALYTICAL LENGTH CHANGE CURVES}

From the results of the previous section, it follows that there is a relation between $\alpha_{0}$, the initial values of $\alpha$, the shear strain, and the 
current value of $\alpha: \alpha=\alpha_{0}-\Upsilon / 2$. Thus, Eq. (8) can be integrated to obtain the length change curve:

$$
\varepsilon=\frac{m}{2(1+m)} \ln f\left(\Upsilon, \alpha_{0}\right),
$$

where

$$
\begin{aligned}
& f\left(Y, \alpha_{0}\right) \\
& \quad=\frac{\left|\cos \left(2 \alpha_{0}-Y\right)-\sin \left(2 \alpha_{0}-Y\right)\right|^{1+1 / m}+\mid \cos \left(2 \alpha_{0}-Y\right)}{\left|\cos 2 \alpha_{0}-\sin 2 \alpha_{0}\right|^{1+1 / m}+\left|\cos 2 \alpha_{0}+\sin 2 \alpha_{0}\right|^{1+1 / m}+\left|2 \sin 2 \alpha_{0}\right|^{1+1 / m}} .
\end{aligned}
$$

For the $m=1$ case:

$$
\varepsilon=\frac{1}{4} \ln \frac{1+2 \sin ^{2}\left(2 \alpha_{0}-\Upsilon\right)}{1+2 \sin ^{2}\left(2 \alpha_{0}\right)} .
$$

In the rate-insensitive limit, the solutions are the following:

$$
\begin{array}{ll}
\varepsilon=\frac{1}{2} \ln \frac{\cos \left(2 \alpha_{0}-Y\right)+\sin \left(2 \alpha_{0}-Y\right)}{\cos 2 \alpha_{0}+\sin 2 \alpha_{0}}, & 0<\left(2 \alpha_{0}-Y\right)<\pi / 8 \\
\varepsilon=\frac{1}{2} \ln \frac{\sin \left(2 \alpha_{0}-Y\right)}{\sin 2 \alpha_{0}}, & \pi / 8<\left(2 \alpha_{0}-Y\right)<3 \pi / 8 \\
\varepsilon=\frac{1}{2} \ln \frac{\cos \left(2 \alpha_{0}-Y\right)-\sin \left(2 \alpha_{0}-Y\right)}{\cos 2 \alpha_{0}-\sin 2 \alpha_{0}}, & 3 \pi / 8<\left(2 \alpha_{0}-Y\right)<\pi / 2
\end{array}
$$

In Figure 5a, length change curves are plotted according to Eq. (16) and Eqs. (17a-c), i.e. for $m=1$ (full lines) and $m=0$ (broken lines), respectively. The measurements of Rose and Stüwe are presented in Figure 5b. As can be seen by comparing Figures $5 \mathrm{a}$ and $5 \mathrm{~b}$, the $m=1$ solution reproduces the experimental length change curves very well. The agreement is least satisfactory for the curves corresponding to $\alpha_{0} \leqq 14^{\circ}$. Because of the symmetries involved in the strain rate ratios (see Figures 2 and 4 and section 4 above), this can be considered to be due to experimental error. Such an explanation is supported by the curve corresponding to $\alpha_{0}=2^{\circ}$ $(m=1$, Figure 5a), which is in good agreement with the measurements corresponding to a stated initial orientation of $\alpha_{0}=7^{\circ}$ (Figure $5 b)$. 


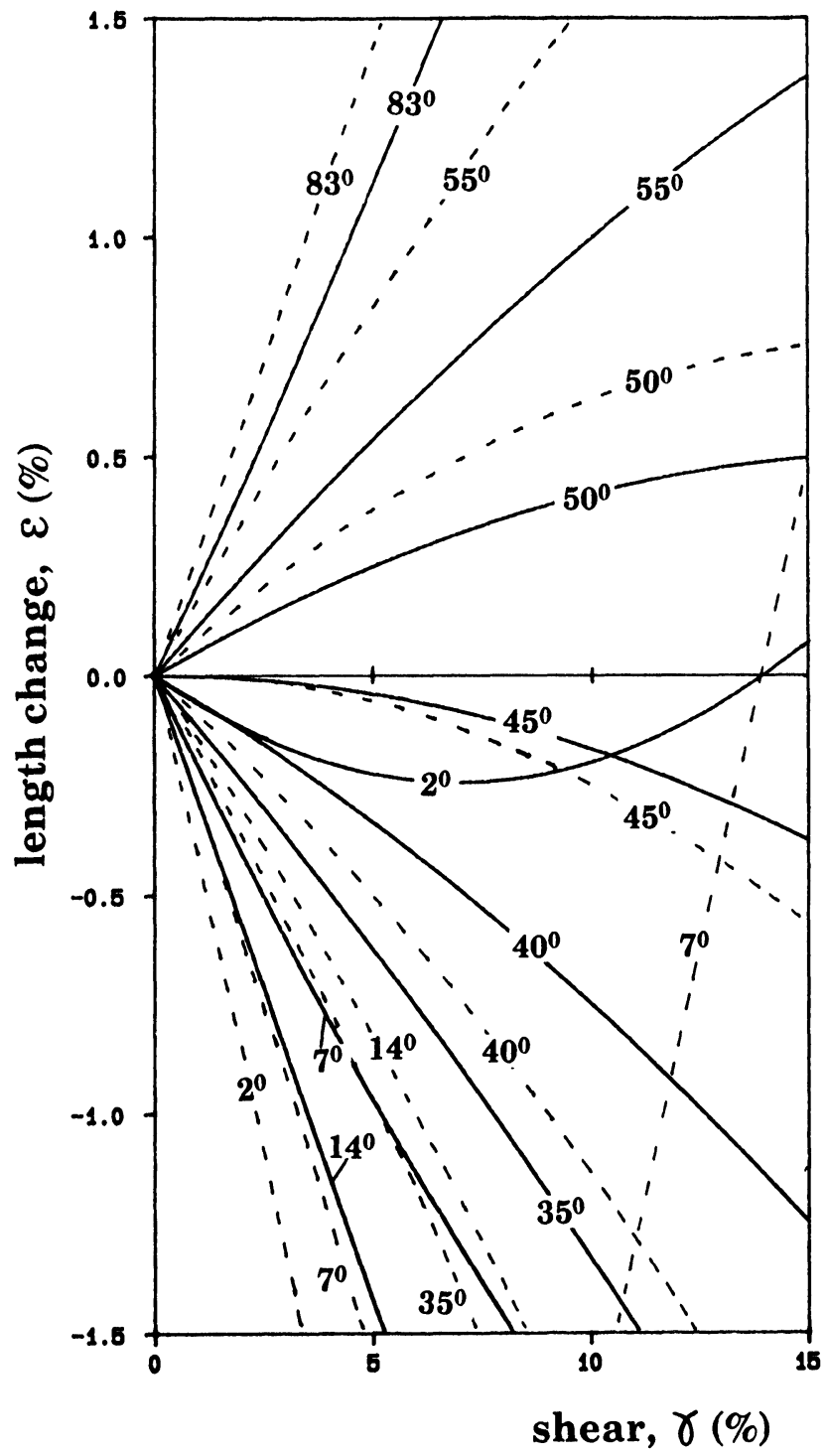

Figure 5(a) Simulated length change curves. The continuous lines correspond to the linear viscous case and the broken lines to the $m=0$ limiting solutions. The numbers indicate the starting orientations. 


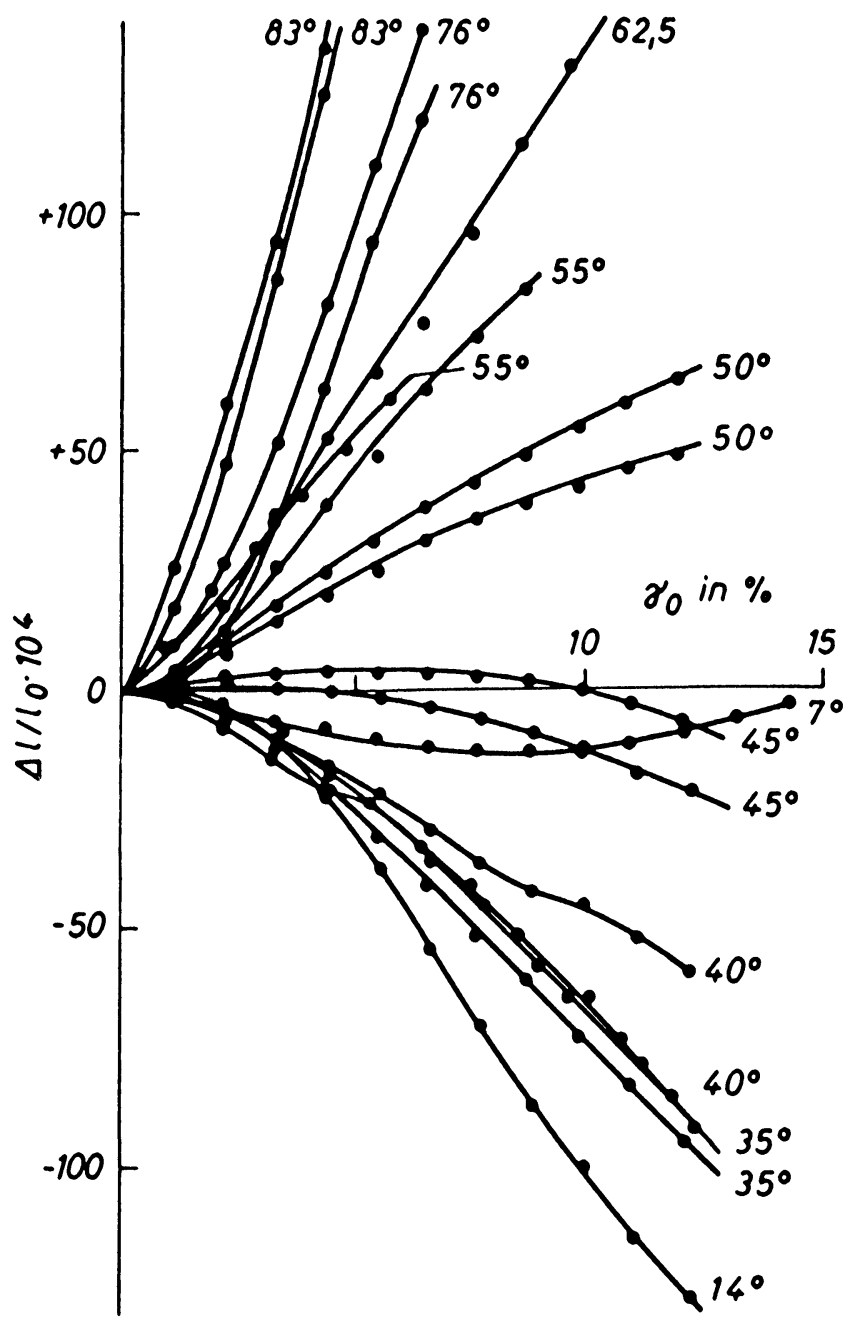

Figure 5(b) Measured length change curves. The spread between the results obtained for pairs of 'identical' samples provides an indication of the accuracy and reproducibility of the experimental technique. 


\section{SUMMARY}

In this paper, a detailed analysis is presented of the free end torsion experiments of Rose and Stüwe (1968). Both rate-sensitive and CMTP methods of analysis have been employed. The simulations are based on the view that the yield surfaces of disoriented single crystals and of polycrystals with a strong cube texture (as in the present case) are similar to those of single crystals, but are more rounded. The two analyses lead to good agreement with the measured length change rate/applied shear rate ratios. One of the advantages of the rate sensitive method is that it permits the calculation of orientation changes during plastic deformation. The latter analysis leads to the result that the plastic spin disappears for the present textures, so that the orientation changes are governed solely by the rigid body rotation. In this way, length change vs. shear strain curves are predicted which are in good agreement with the experimental results.

\section{Acknowledgements}

The authors are indebted to Dr. M. Darrieulat of the École des Mines de St. Etienne and to Prof. S. C. Shrivastava for numerous stimulating discussions. They are thankful to the Canadian Steel Industry Research Association and the Ministry of Education of Quebec (FCAR program) for financial support. LST acknowledges with gratitude the International Exchange Award granted by the Natural Sciences and Engineering Research Council of Canada as well as the period of sabbatical leave accorded by the Eötvös University in Hungary.

\section{References}

Aifantis, E. C. (1987). Int. J. Plasticity 3, 211.

Canova, G. R., Molinari, A., Fressengeas, C. and Kocks, U. F. (1988). Acta. Metall., 36, 1961.

Harren, S., Lowe, T., Asaro, R. J. and Needleman, A. (in press). Phil. Trans. Royal. Soc. London.

Lequeu, Ph., Gilormini, P., Montheillet, F., Bacroix, B. and Jonas, J. J. (1987a). Acta Metall 35, 439.

Lequeu, Ph., Gilormini, P., Montheillet, F., Bacroix, B. and Jonas, J. J. (1987b). Acta Metall 35, 1159. 
Lequeu, Ph., Jonas, J. J. (1988). Metall. Trans. 19A, 105.

Lequeu, Ph., Montheillet, F. and Jonas, J. J. (1985) Proc. 7th. Int. Conf. on the Strength of Metals and Alloys, Eds. H. J. McQueen, J. P. Bailon, J. I. Dickson, J. J. Jonas and M. G. Akben, Montreal p. 269.

Montheillet, F., Gilormini, P. and Jonas, J. J. (1985). Acta Metall. 33, 705.

Rose, W. and Stüwe, H. P. (1968). Z. Metallkde 59, 396.

Semiatin, S. L., Lahoti, G. D. and Jonas, J. J. (1985). ASM Metals Handbook, Vol 8, 9th Ed. American Society for Metals, Metals Park Ohio p. 181.

Toth, L. S., Gilormini, P. and Jonas, J. J. (1988). Acta Metall. 36, 3077.

Tóth, L. S., Jonas, J. J., Gilormini, P. and Bacroix, B. (in press). Int. J. Plasticity. 